OPEN ACCESS

Edited by:

Vin-Cent Wu,

National Taiwan University, Taiwan

Reviewed by:

Marloes Dekker Nitert

The University of Queensland,

Australia

Silvia Monticone

University of Turin, Italy

Shenghui Li,

China Agricultural University, China

${ }^{*}$ Correspondence:

Yuchun Zhu

mmaalleee@126.com

Liang Zhou

zhouliang5678@foxmail.com

${ }^{t}$ These authors have contributed equally to this work and

share first authorship

Specialty section:

This article was submitted to

Neuroendocrine Science,

a section of the journal

Frontiers in Endocrinology

Received: 15 February 2021 Accepted: 28 July 2021

Published: 17 August 2021

Citation:

Liu Y, Jiang Q, Liu Z, Shen S,

Ai J, Zhu Y and Zhou L (2021)

Alteration of Gut Microbiota Relates

to Metabolic Disorders in Primary

Aldosteronism Patients.

Front. Endocrinol. 12:667951.

doi: 10.3389/fendo.2021.667951

\section{Alteration of Gut Microbiota Relates to Metabolic Disorders in Primary Aldosteronism Patients}

\author{
Yu Liu ${ }^{\dagger}$, Qingyao Jiang ${ }^{\dagger}$, Zhihong Liu, Sikui Shen, Jianzhong Ai, Yuchun Zhu* \\ and Liang Zhou*
}

Department of Urology, Institute of Urology (Laboratory of Reconstructive Urology), West China Hospital, Sichuan University, Chengdu, China

Purpose: This study aimed to determine the relationships among gut microbiota, primary aldosteronism (PA), and related metabolic disorders.

Methods: The study enrolled 13 PA patients, 26 sex-matched primary hypertension patients, and 26 sex-matched healthy controls. Demographic and clinical characteristics such as age, body mass index (BMI), blood aldosterone-renin ratio, blood potassium, blood glucose, blood lipid parameters, and history of diabetes mellitus (DM) were compared between the three groups. The gut microbiota of each participant was examined by $16 S$ rRNA gene sequencing. Spearman correlation analysis was performed to demonstrate the relationship between gut microbiota and clinical characteristics.

Results: $\mathrm{BMI}$ and the percentage of DM in PA patients were higher than those in healthy controls $(p<0.05)$, but not higher than those in primary hypertension patients $(p>0.05)$. The gut microbiota of healthy controls and primary hypertension patients had a higher alpha diversity level than that of PA patients. PA patients had fewer short-chain fatty acid (SCFA)-producing genera (Prevotella, Blautia, Coprococcus, Anaerostipes, and Ruminococcus) and more inflammation-associated genera (Megamonas, Sutterella, and Streptococcus) than healthy controls $(p<0.05)$. The gut microbiota of PA patients was more inclined to encode microbial pathways involved in sugar metabolism, such as starch and sucrose metabolism and fructose and mannose metabolism. Blood potassium was negatively correlated with the relative abundance of Romboutsia ( $R=-0.364, q=0.023)$. Diastolic blood pressure (DBP) was positively correlated with Romboutsia $(R=0.386$, $q=0.015)$. Systolic blood pressure (SBP) was negatively correlated with Blautia $(R=-0.349, q=0.030)$.

Conclusions: The alteration of gut microbiota in PA patients, especially bacteria and pathways involved in inflammation, SCFAs, and sugar metabolism, may be associated with chronic metabolic disorders. 


\section{INTRODUCTION}

Primary aldosteronism (PA) is the most common cause of secondary hypertension (1). PA is frequently poorly diagnosed and treated, leading to aldosterone-specific morbidity and mortality. Many observational studies have reported an increased prevalence of metabolic complications [such as diabetes mellitus (DM) and obesity] among PA patients $(2,3)$. Hyperaldosteronemia, hypokalemia, and cortisol secretion are regarded as risk factors for metabolic disorders (4). However, a clear pathophysiological interrelationship linking the two entities has yet to be established.

The gut microbiome is part of a complex ecosystem. Many studies have shown that the gut microbiota and its metabolites are involved in some metabolic diseases, such as metabolic syndrome, DM, and obesity (5-8). Since the reasons for high prevalence of metabolic diseases in PA patients have not been well described, it will be interesting to explore the interaction between PA-induced metabolic disorders and independent microbial characteristics. To address this problem, we conducted a case-control study with 65 participants in which we analyzed gut microbiota to establish the intestinal microbial profiles of PA patients. We aimed to determine the relationship between gut microbiota, PA, and related metabolic diseases.

\section{MATERIALS AND METHODS}

\section{Study Population}

A case-control study was performed at West China Hospital, Sichuan University in China from September 2019 to December 2019. We recruited PA patients, primary hypertension patients, and healthy controls. PA patients (PA group) were primarily diagnosed by the aldosterone-renin ratio (cutoff value $=30$ ), and then confirmed by an intravenous saline load test (cutoff value of postsaline aldosterone $=5 \mathrm{ng} / \mathrm{dl}$ ) and a captopril challenge test (cutoff value of postcaptopril aldosterone $=11 \mathrm{ng} / \mathrm{dl}$ ). Abdominal computed tomography was performed to determine the location of the adrenal nodules. Adrenal venous sampling was performed to preoperatively distinguish unilateral and bilateral PA. Primary hypertension patients (hypertension group) were defined as patients with hypertension who had systolic blood pressure (SBP) $\geq 140 \mathrm{mmHg}$ or diastolic blood pressure (DBP) $\geq 90 \mathrm{mmHg}$. Blood cortisol, catecholamine and renin-angiotensin-aldosterone, thyroid hormone, thyroid-stimulating hormone, and parathyroid hormone were examined to verify that they did not have endocrine hypertension. Acromegaly and obstructive sleep apnea were also considered if patients reported related symptoms (1). Healthy controls (control group) were defined as participants without hypertension, with SBP $\leq 139 \mathrm{mmHg}$ and DBP $\leq 89 \mathrm{mmHg}$. Blood pressure was measured in a sitting position by nurses or physicians. Three readings were recorded at 5-min intervals with a random-zero mercury column sphygmomanometer, and the ;average was taken as the final measurement. All PA patients received laparoscopic adrenalectomy, with the pathological specimen confirmed as cortical adenoma.
This study was approved by the West China Hospital, Sichuan University Medical Research Ethics Committee (IRB approval number 2018182), and informed consent was obtained from each participant.

Participants were excluded if they had the following conditions: the use of antibiotics within 3 months before fecal sampling, inflammatory bowel disease, irritable bowel syndrome, digestive tract infection, tumors of the digestive system, intestinal surgery, and recurrent diarrhea or constipation within 1 month before stool sample collection.

\section{Collection of Demographic and Clinical Characteristics}

A questionnaire was designed to query the demographic and clinical information of participants, including sex, age, and history of smoking and drinking. Weight and height were measured by instruments. Body mass index (BMI) was calculated by dividing weight in kilograms by the square of height in meters. Blood pressure, blood aldosterone-renin ratio, blood potassium, blood glucose, blood lipid parameters [triglyceride, cholesterol, high-density lipoprotein (HDL) and low-density lipoprotein (LDL)], and history of DM were obtained from medical records. Fecal samples of the study participants were collected at inpatient or outpatient departments when PA and primary hypertension patients visited our hospital. We asked healthy controls from the health examination center of our hospital to provide stool samples voluntarily. The samples were immediately frozen in liquid nitrogen and subsequently stored at $-80^{\circ} \mathrm{C}$ until analysis.

\section{Analyses of Gut Microbiota}

Microbial DNA was extracted from fecal samples using a QIAamp Fast DNA Stool Mini Kit. Negative controls were conducted using sterile water to exclude any possible contamination. The V3-V4 regions of the 16S rRNA gene were amplified with primers $338 \mathrm{~F}$ (5'-ACTCCTACGGGAG GCAGCAG-3') and 806R (5'-GGACTACHVGGGTWT CTAAT $\left.-3^{\prime}\right)$. We constructed a library using the NEXTFLEX Rapid DNA-Seq Kit. Amplicons were pooled in equimolar amounts and paired-end sequenced $(2 \times 300)$ on an Illumina MiSeq platform. After demultiplexing, the resulting sequences were merged by FLASH (version 1.2.11) (9) and quality filtered with fastp (version 0.19.6) (10). Then the sequences were denoised using the DADA2 (11) plugin in QIIME2 (version 2020.2) (12). The denoised sequences are called amplicon sequence variants (ASVs). Taxonomic assignment of ASVs was performed with the SILVA $16 \mathrm{~S}$ rRNA gene database (version 138).

Based on the sequencing data, the coverage index was first calculated to determine if the sequencing depth covered the whole bacterial diversity. The higher the coverage index is, the higher the probability of the sequence being detected. We compared the alpha diversity indices, such as the Shannon and Simpson indices between the control, hypertension, and PA groups. A higher Shannon index and a lower Simpson index 
indicate a higher richness and evenness of the gut microbiota. Interindividual variability (beta diversity) among these three groups was also evaluated with principal coordinates analysis (PCoA) by Bray-Curtis distance, the permutational multivariate analysis of variance (PERMANOVA) test, and the analysis of similarities (ANOSIM) test. The linear discriminant analysis (LDA) effect size (LEfSe) method was used to identify differentially abundant bacteria among the three groups (13). The Kyoto Encyclopedia of Genes and Genomes (KEGG) metabolic pathways were predicted by PICRUSt2 (14). A random forest algorithm was used to determine which bacteria had key roles in distinguishing PA patients from primary hypertension patients and healthy controls. Spearman correlation analysis was performed to demonstrate the relationship between gut microbiota and clinical characteristics.

\section{Statistical Analysis}

Qualitative parameters are presented as numbers and percentages. Quantitative parameters were reported as the mean and standard deviation if they were symmetrically distributed. Otherwise, they are shown as the median and interquartile range. Fisher's exact test was used to examine qualitative parameters. Student's $t$-test, analysis of variance (ANOVA), Mann-Whitney $U$ test, and Kruskal-Wallis test were applied for testing quantitative parameters. We performed all the statistical analyses in $\mathrm{R}$ (version 3.6.3) ( $\mathrm{R}$ Project for Statistical Computing, www.rproject.org).

\section{RESULTS}

\section{General Characteristics of Healthy Controls, Primary Hypertension Patients, and PA Patients}

A total of 65 participants were enrolled in our study, consisting of 13 PA patients, 26 sex-matched primary hypertension patients, and 26 sex-matched healthy controls (Table 1). The mean ages of the control group (50.1 years) and the PA group (46.4 years) were not significantly different $(p=0.196)$, while they were both lower than that of the hypertension group (56.9 years) $(p<0.05)$. BMI was higher in PA patients $(24.5)$ than in healthy controls (23.5) ( $p=0.043)$, while it was not significantly different between the PA group and the hypertension group $(p=0.126)$. PA patients had higher DBP than primary hypertension patients $(p<0.001)$, while their SBPs were not significantly different $(p=0.151)$. The blood potassium level of the PA group was lower than those of the hypertension and PA groups $(p<0.001)$. More PA patients had DM than healthy controls $(30.8 \%$ vs. $3.8 \%, p=0.018)$. However, the percentages of DM were not significantly different between PA patients and primary hypertension patients $(30.8 \%$ vs. $26.9 \%$, $p=0.801)$. History of smoking and drinking, blood glucose, and blood lipid parameters were not different between the three groups $(p>0.05)$.

PA patients used many types of antihypertensive drugs before surgery, including angiotensin-converting enzyme inhibitors,

TABLE 1 | Demographic and clinical characteristics of study participants in control, primary hypertension, and PA patients.

\begin{tabular}{|c|c|c|c|c|c|c|c|}
\hline Parameters & $\mathrm{PA}(n=13)$ & $\begin{array}{c}\text { Primary } \\
\text { hypertension } \\
(n=26)\end{array}$ & $\begin{array}{l}\text { Control } \\
(n=26)\end{array}$ & $\begin{array}{c}p \text {-value } \\
\text { (PA vs. primary } \\
\text { hypertension vs. } \\
\text { control) }\end{array}$ & $\begin{array}{l}\text { p-value } \\
\text { (PA vs. } \\
\text { control) }\end{array}$ & $\begin{array}{c}\text { p-value } \\
\text { (PA vs. } \\
\text { primary } \\
\text { hypertension) }\end{array}$ & $\begin{array}{c}\text { p-value } \\
\text { (primary } \\
\text { hypertension vs. } \\
\text { control) }\end{array}$ \\
\hline Sex & & & & $1.000(\mathrm{a})$ & $1.000(\mathrm{a})$ & $1.000(\mathrm{a})$ & $1.000(\mathrm{a})$ \\
\hline Male & 8 (61.5\%) & $16(61.5 \%)$ & $16(61.5 \%)$ & & & & \\
\hline Female & 5 (38.5\%) & $10(38.5 \%)$ & $10(38.5 \%)$ & & & & \\
\hline Age (years) & $46.4(11.8)$ & $56.9(6.9)$ & $50.1(6.0)$ & $<0.001(\mathrm{~b})$ & 0.196 (b) & 0.001 (b) & $<0.001$ (b) \\
\hline $\mathrm{BMI}\left(\mathrm{kg} / \mathrm{m}^{2}\right)$ & $24.5(6.4)$ & $24.1(5.1)$ & $23.5(3.5)$ & $0.124(\mathrm{c})$ & 0.043 (c) & $0.126(\mathrm{c})$ & $0.558(\mathrm{c})$ \\
\hline $\mathrm{SBP}(\mathrm{mmHg})$ & $147.9(13.8)$ & $140.9(14.3)$ & $122.7(13.2)$ & $<0.001$ (b) & $<0.001$ (b) & 0.151 (b) & $<0.001$ (b) \\
\hline $\mathrm{DBP}(\mathrm{mmHg})$ & $99.2(12.2)$ & $83.2(8.2)$ & $77.7(9.6)$ & $<0.001$ (b) & $<0.001$ (b) & $<0.001$ (b) & 0.031 (b) \\
\hline Aldosterone-renin ratio & $426.2(709.3)$ & $6.1(7.6)$ & - & - & - & $<0.001$ (c) & - \\
\hline Blood potassium (mmol/L) & $3.20(0.22)$ & $3.93(0.22)$ & $4.34(0.37)$ & $<0.001$ (b) & $<0.001$ (b) & $<0.001$ (b) & $<0.001$ (b) \\
\hline Blood glucose (mmol/L) & $5.42(1.33)$ & $5.35(0.95)$ & $4.88(0.65)$ & 0.053 (c) & $0.036(\mathrm{c})$ & 0.964 (c) & 0.019 (c) \\
\hline Triglyceride (mmol/L) & $0.96(2.18)$ & $1.45(0.96)$ & $1.41(0.41)$ & $0.231(\mathrm{c})$ & $0.126(c)$ & $0.136(\mathrm{c})$ & $0.641(\mathrm{c})$ \\
\hline Cholesterol (mmol/L) & $4.35(0.81)$ & $4.41(1.02)$ & $4.92(0.96)$ & 0.101 (b) & 0.077 (b) & 0.864 (b) & $0.070(\mathrm{~b})$ \\
\hline $\mathrm{HDL}(\mathrm{mmol} / \mathrm{L})$ & $1.27(0.33)$ & $1.25(0.28)$ & $1.26(0.23)$ & 0.973 (b) & 0.924 (b) & 0.839 (b) & 0.868 (b) \\
\hline LDL (mmol/L) & $2.62(0.64)$ & $2.54(0.89)$ & $2.64(0.81)$ & 0.899 (b) & $0.939(b)$ & $0.772(b)$ & $0.671(b)$ \\
\hline DM & & & & 0.036 (a) & 0.035 (a) & $1.000(\mathrm{a})$ & $0.050(\mathrm{a})$ \\
\hline Yes & $4(30.8 \%)$ & 7 (26.9\%) & $1(3.8 \%)$ & & & & \\
\hline No & 9 (69.2\%) & $19(73.1 \%)$ & 25 (96.2\%) & & & & \\
\hline Smoking & & & & $0.835(\mathrm{a})$ & $0.719(\mathrm{a})$ & $0.714(\mathrm{a})$ & $1.000(\mathrm{a})$ \\
\hline Yes & 3 (23.1\%) & 9 (34.6\%) & $8(30.8 \%)$ & & & & \\
\hline No & $10(76.9 \%)$ & $17(65.4 \%)$ & $18(69.2 \%)$ & & & & \\
\hline Drinking & & & & $1.000(\mathrm{a})$ & $1.000(\mathrm{a})$ & $1.000(\mathrm{a})$ & $1.000(\mathrm{a})$ \\
\hline Yes & 4 (30.8\%) & 9 (34.6\%) & $8(30.8 \%)$ & & & & \\
\hline No & 9 (69.2\%) & 17 (65.4\%) & 18 (69.2\%) & & & & \\
\hline
\end{tabular}

(a) Fisher's exact test, (b) Student's t-test, (c) Mann-Whitney $U$ test.

$P A$, primary aldosteronism; BMI, body mass index; SBP, systolic blood pressure; DBP, diastolic blood pressure; HDL, high-density lipoprotein; LDL, low-density lipoprotein; DM, diabetes mellitus. 
angiotensin receptor blockers, alpha-blockers, beta-blockers, calcium channel blockers, diuretics, and mineralocorticoid receptor antagonists, where only one PA patient used spironolactone. The aldosterone-renin ratio of PA patients was much higher than that of primary hypertension patients $(426.2$ vs. 6.1, $p<0.001)$.

\section{Diversity of the Gut Microbiota in Healthy Controls, Primary Hypertension Patients, and PA Patients}

Healthy controls and primary hypertension patients had a higher Shannon index and a lower Simpson index than PA patients $(p<0.001)$. This result indicated that the within-sample alpha diversity level of the gut microbiota of PA patients was lower than that of healthy controls and primary hypertension patients (Figures 1A, B and Supplementary Table 1). The rarefaction curves reached the saturation plateau, and the coverage index ranged from 0.9975 to 0.9998 , which indicated that the sequencing depth for the Sobs index was sufficient (Figure 1C).
PCoA by Bray-Curtis distance revealed an asymmetrical distribution of gut microbiota composition between the control, hypertension, and PA groups (Figure 1D). The ANOSIM test $(R=0.599, p=0.001)$ and PERMANOVA test $\left(R^{2}=0.093, p=0.001\right)$ both verified that there were significant differences in bacterial composition between the three groups.

\section{Taxonomic Analysis of Microbiota Composition Between Healthy Controls, Primary Hypertension Patients, and PA Patients}

At the phylum level (Figure 2A), Firmicutes (mean relative abundance, 62.4\%) was the most abundant bacteria in all analyzed samples, followed by Bacteroidota (22.9\%), Proteobacteria (6.7\%), and Actinobacteria (4.7\%). The Firmicutes/Bacteroidetes ratio of the gut microbiota was higher in healthy controls (3.23) than in primary hypertension patients (2.45) and PA patients (2.48). At the genus level (Figure 2B), Bacteroides was the most common genus, whose percentage in
A

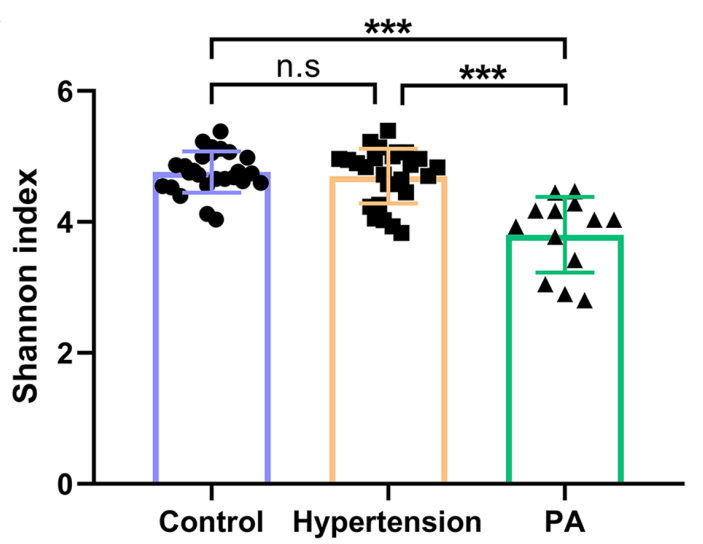

C

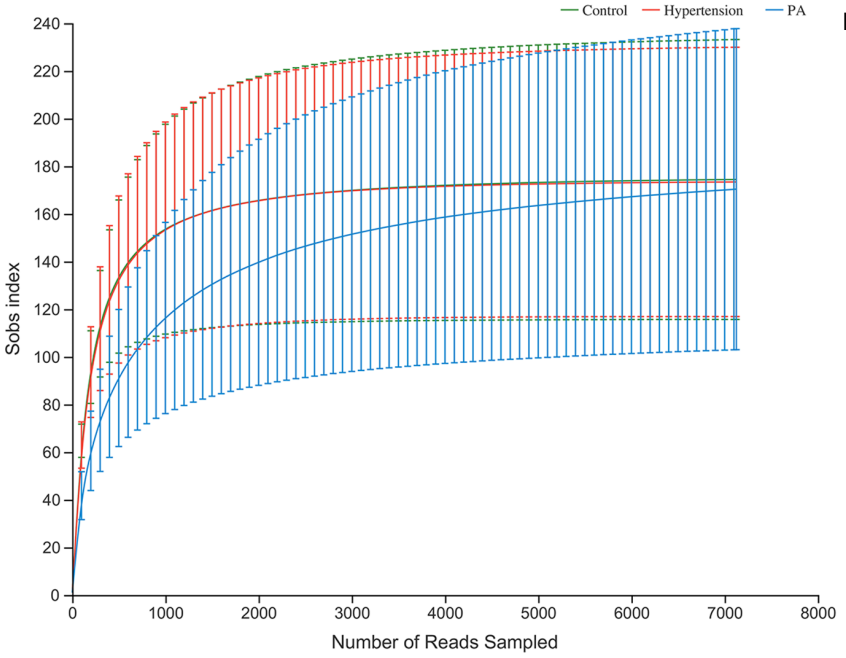

B

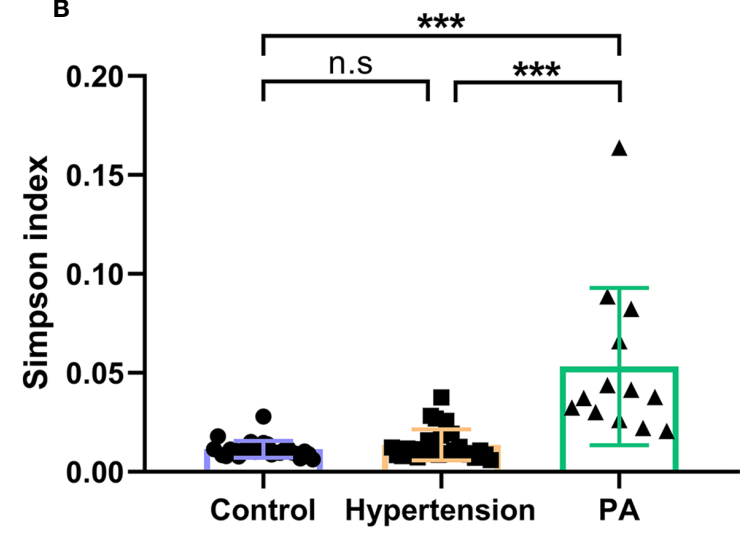

D

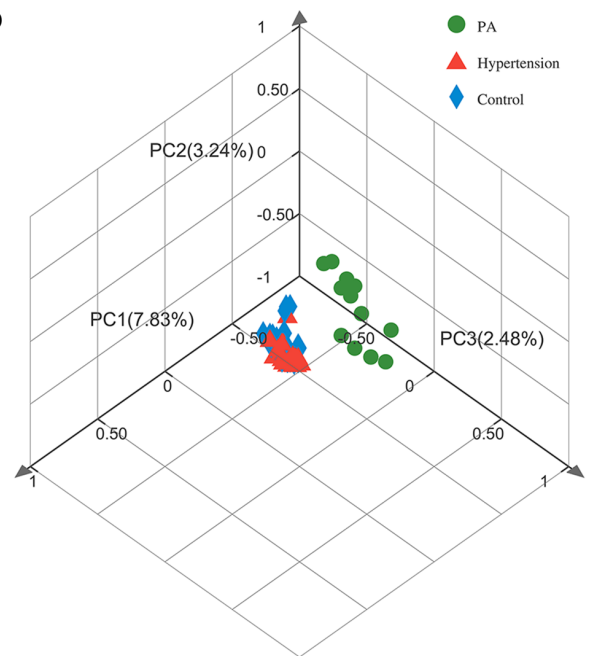

FIGURE 1 | Diversity of the gut microbiota in primary aldosteronism (PA) patients, primary hypertension patients, and healthy controls. (A) Shannon index and (B) Simpson index of gut microbiota. (C) Rarefaction curves of all the samples in the three groups. The horizontal axis shows the number of sequences obtained by sequencing the 16S rRNA gene. The vertical axis shows the number of genera. (D) PCoA of gut microbiota by Bray-Curtis distance. ${ }^{\star \star \star} p<0.001$. n.s, not significant. 
A

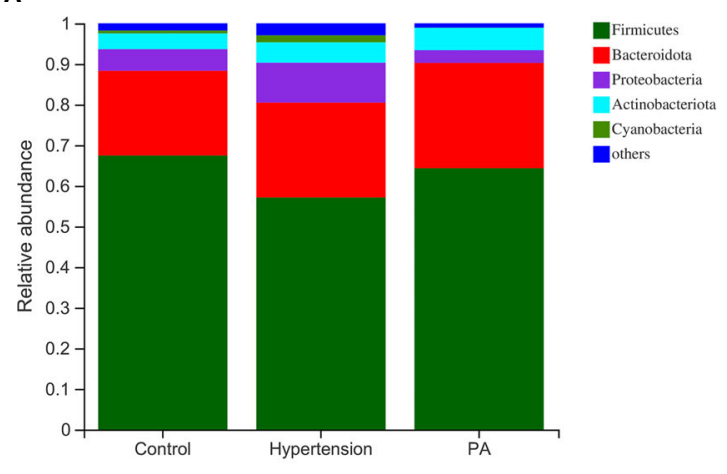

B

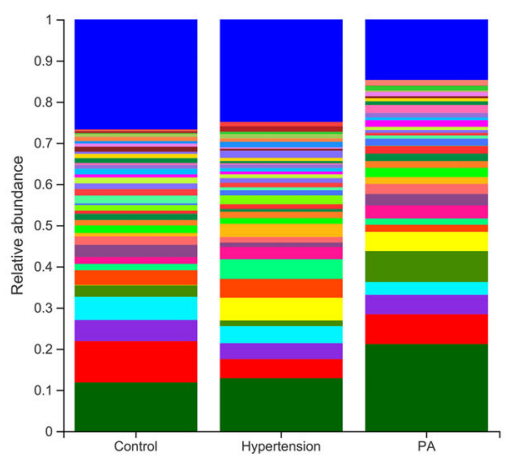

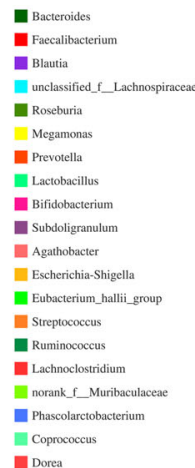

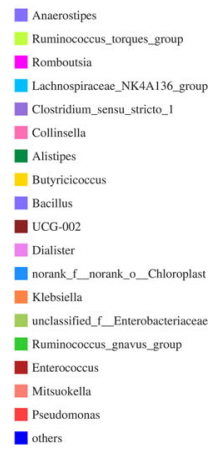

FIGURE 2 | Distribution of gut microbiota between primary aldosteronism (PA) patients, primary hypertension patients, and healthy controls. Relative abundances of the major bacteria at the $\mathbf{( A )}$ phylum and $\mathbf{( B )}$ genus levels.

the gut microbiota was much higher in PA patients $(21.1 \%)$ than in primary hypertension patients $(12.9 \%, p=0.043)$ and healthy controls $(11.8 \%, p=0.032)$. The second most common genus was Faecalibacterium, whose percentage in the gut microbiota was higher in healthy controls $(10.0 \%)$ than in primary hypertension patients $(4.6 \%, p=0.011)$ and PA patients $(7.3 \%, p=0.038)$.

LEfSe analysis was used to determine the bacterial genera with significant differences between healthy controls, primary hypertension patients, and PA patients. Compared with PA patients, there were 28 and 35 genera with significantly different relative abundances in healthy controls and primary hypertension patients, respectively (Figures 3A, B). For example, PA patients had less Prevotella $(p=0.018)$, Blautia $(p=0.008)$, Lactobacillus ( $p<0.003)$, Coprococcus $(p=0.007)$, Eubacterium eligens group ( $p=0.004)$, Anaerostipes $(p=0.006)$, Ruminococcus torques group $(p<0.032)$, and Enterococcus $(p=0.006)$ and more Bacteroides $(p=0.032)$, Megamonas $(p=0.001)$, and Sutterella ( $p=0.001)$ than healthy controls. Similarly, the relative abundances of Lactobacillus ( $p<0.001)$, Prevotella $(p<0.001)$, Weissella $(p<0.001)$, Lactococcus $(p<0.001)$, and Akkermansia $(p<0.001)$ were higher in primary hypertension patients, while those of Roseburia $(p=0.003)$, Streptococcus $(p=0.024)$, Paraprevotella ( $p=0.011)$, and Sutterella $(p=0.016)$ were higher in PA patients. Genera with significant differences between the control and hypertension groups are shown in Supplementary Figure 1.

We performed a Bray-Curtis distance-based redundancy analysis (dbRDA) to determine the additional effects of age, $\mathrm{BMI}$, and DM on the gut microbiota (Figure 4). The envfit function test showed that age $(p=0.001)$, BMI $(p=0.001)$, and DM $(p=0.001)$ were significant explanatory variables (Supplementary Table 2).

To eliminate the possible confounding effects of DM and BMI, we excluded participants with DM. BMI and blood glucose were not significantly different between healthy controls, primary hypertension patients, and PA patients after removing DM patients $(p>0.05)$ (Supplementary Table 3$)$. LEfSe analysis revealed that the genera with significant differences between the three groups were similar to those before deleting participants with DM (Supplementary Figure 2). After deleting DM patients, the relative abundance of Eubacterium ( $p=0.017$ ) was higher in PA patients than in healthy controls. PA patients also had more Bacteroides $(p=0.013)$, Phascolarctobacterium $(p=0.024)$, Moryella ( $p=0.009$ ), and Eubacterium fissicatena group $(p=0.036)$ than primary hypertension patients after deleting DM patients.

\section{Metabolic Function of Gut Microbiota}

To evaluate the gene information of gut microbiota, metabolic predictions were achieved using PICRUSt2. The relative abundance of metabolic pathways between the three groups was compared by the Wilcoxon rank sum test. The BenjaminiHochberg false discovery rate (FDR)-adjusted $p$-values were also calculated. Compared with healthy controls, PA patients had more lipopolysaccharide biosynthesis (FDR-adjusted $p$ value $=0.017$, galactose metabolism (FDR-adjusted $p$ value $=0.001)$, pentose and glucuronate interconversions (FDR-adjusted $p$-value $=0.004)$, amino sugar and nucleotide sugar metabolism $($ FDR-adjusted $p$-value $=0.001)$, fructose and mannose metabolism (FDR-adjusted $p$-value $=0.004$ ), starch and sucrose metabolism (FDR-adjusted $p$-value $=0.002$ ), and arginine and proline metabolism (FDR-adjusted $p$-value $=0.006$ ) (Supplementary Table 4). The relative abundance of tryptophan metabolism (FDR-adjusted $p$-value $=0.010)$ was higher in primary hypertension patients, while those of galactose metabolism (FDRadjusted $p$-value $=0.005)$, insulin resistance $($ FDR-adjusted $p$-value $=0.001$ ), starch and sucrose metabolism (FDR-adjusted $p$-value $=0.003)$, amino sugar and nucleotide sugar metabolism (FDR-adjusted $p$-value $=0.011)$, pentose and glucuronate interconversions (FDR-adjusted $p$-value $=0.018$ ), and insulin signaling pathway (FDR-adjusted $p$-value $=0.041$ ) were higher in PA patients (Supplementary Table 5). Supplementary Table 6 shows the metabolic pathways with significant differences between the control and hypertension groups. 
A

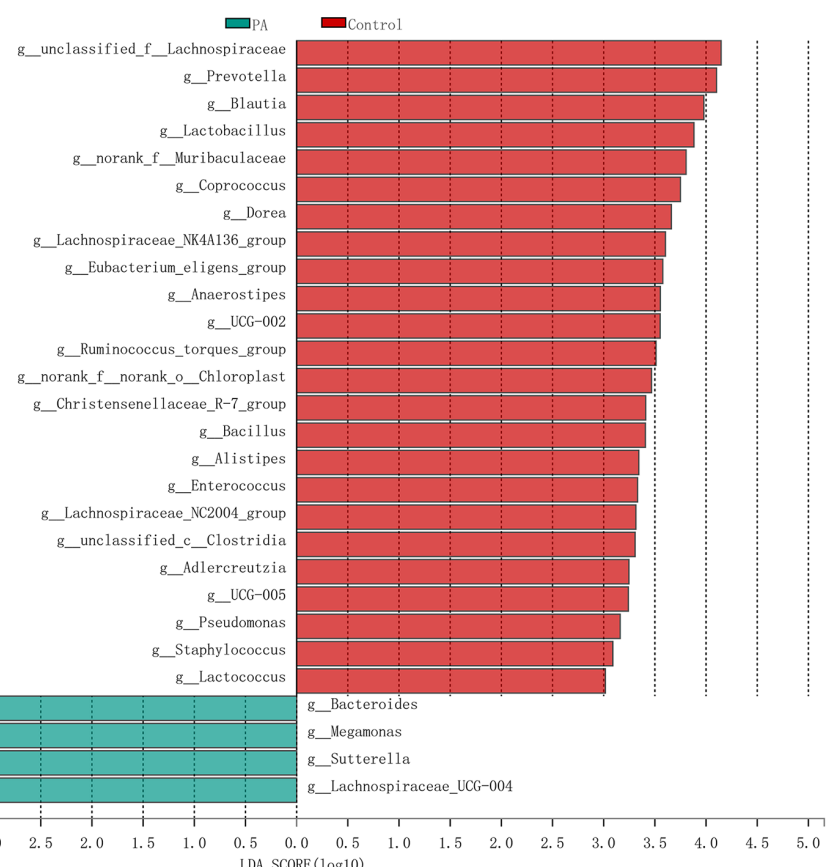

B

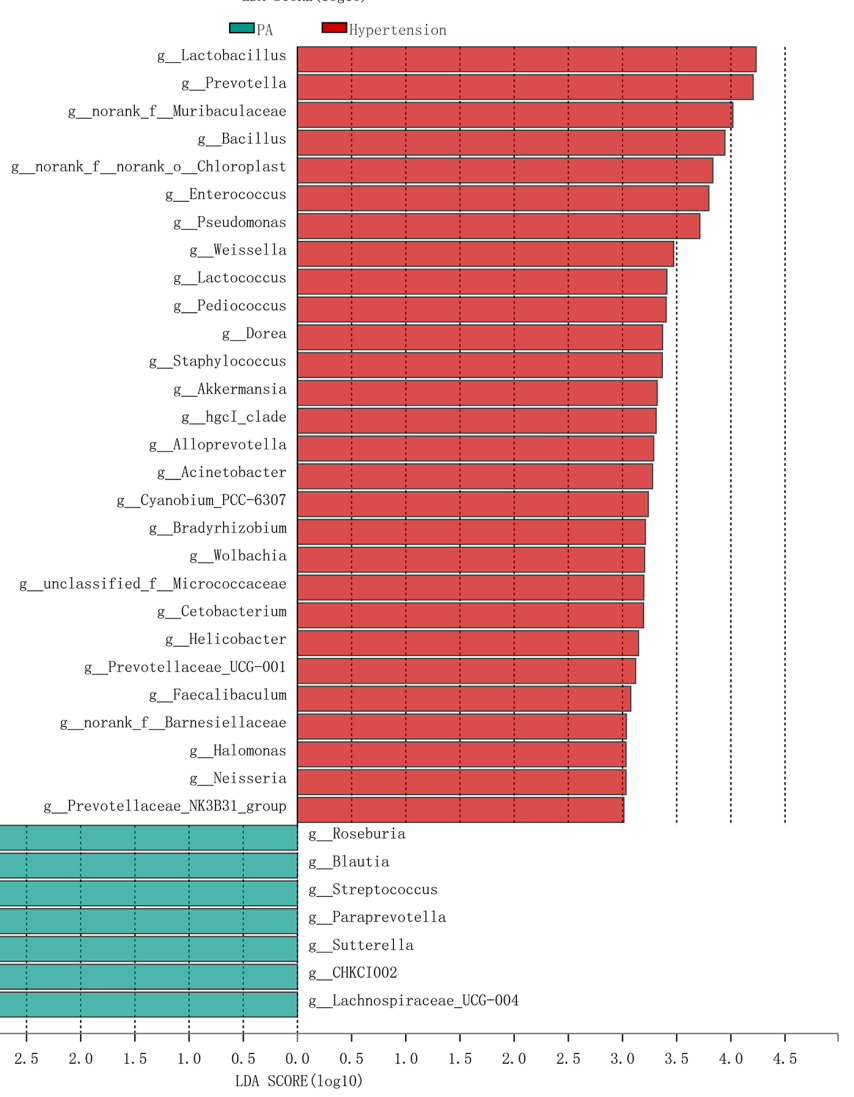

FIGURE 3 | Differences in intestinal bacteria between primary aldosteronism (PA) patients, primary hypertension patients, and healthy controls. (A) Significantly different genera between PA patients and healthy controls. Red bars are genera with higher relative abundances in healthy controls. Green bars are genera with higher relative abundances in PA patients. (B) Significantly different genera between PA patients and primary hypertension patients. Red bars are genera with higher relative abundances in primary hypertension patients. Green bars are genera with higher relative abundances in PA patients. 


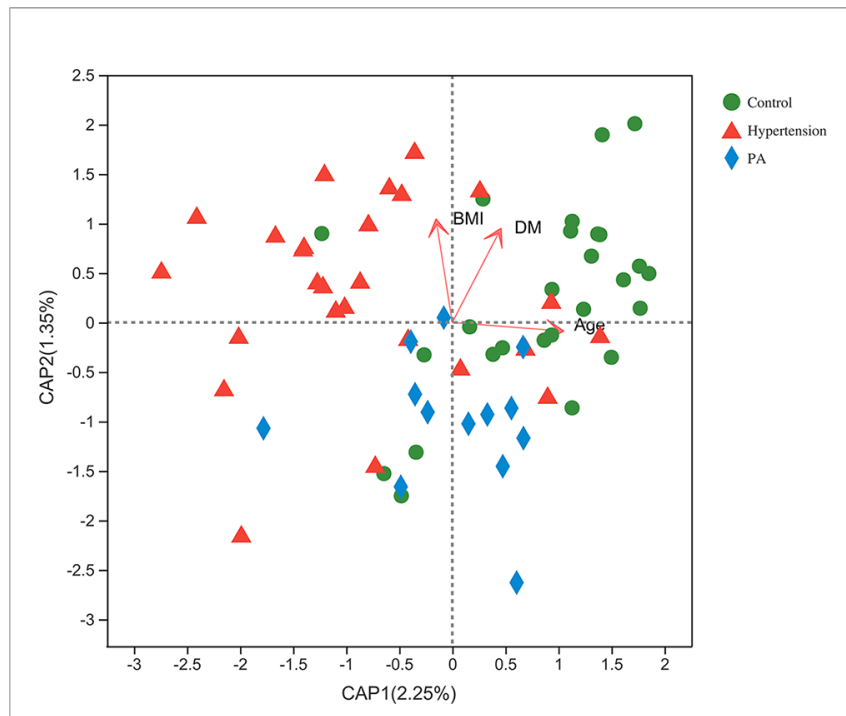

FIGURE 4 | Bray-Curtis distance-based redundancy analysis (dbRDA) of the gut microbiota and explanatory factors in primary aldosteronism (PA) patients, primary hypertension patients, and healthy controls.

\section{Bacteria Distinguishing PA Patients From Primary Hypertension Patients and Healthy Controls}

We used random forest algorithm to determine which bacteria had key roles in distinguishing PA patients from healthy controls. Figure 5A shows the top 30 genera with the greatest importance, some of which were also screened out by LEfSe analysis, such as Blautia, Anaerostipes, Megamonas, and Lactobacillus. The area under the curve (AUC) of the receiver operating characteristic curve was 0.8173 using the random forest model in combination with leave-one-out crossvalidation. Similarly, Figure 5B presents the top 30 genera with the greatest importance in distinguishing PA patients from primary hypertension patients. The following genera were also detected in the LEfSe analysis: Pseudomonas, Enterococcus, Akkermansia, Weissella, and Pediococcus. The AUC of the receiver operating characteristic curve was 0.9231.

\section{Association Between Clinical Characteristics and Gut Microbiota}

Heatmap analysis (Figure 6A) showed that the bacteria with lower relative abundances in the PA group than in the hypertension group were positively correlated with blood potassium and negatively correlated with SBP and DBP. We specifically drew scatter plot graphs of genera with high percentages in the gut microbiota (Figures 6B-D). Blood potassium was negatively correlated with the relative abundance of Romboutsia $(R=-0.364, q=0.023)$. In addition, DBP was positively correlated with Romboutsia $(R=0.386$, $q=0.015)$. SBP was negatively correlated with Blautia $(R=-0.349, q=0.030)$.

\section{DISCUSSION}

This study is the first to demonstrate the characteristics of the gut microbiota in PA patients. The gut microbiota has been reported to be associated with metabolic diseases, such as DM and obesity $(6,7)$. DM and obesity are more common in PA patients than in healthy people or primary hypertension patients $(2,3)$. Our study showed that DM was more common in PA patients than in healthy controls. The BMI of PA patients was higher than that of healthy controls. In addition, the composition of the gut
A

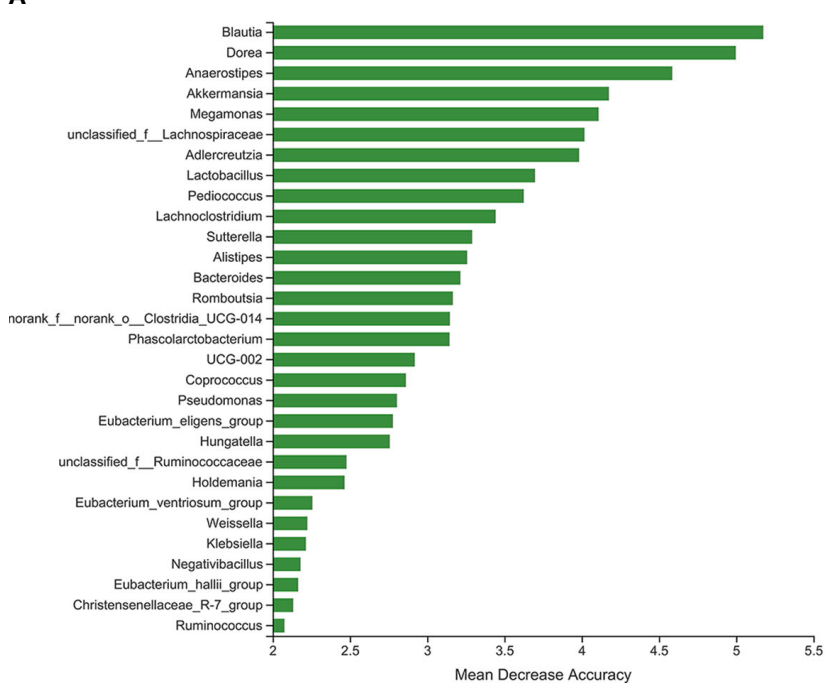

B

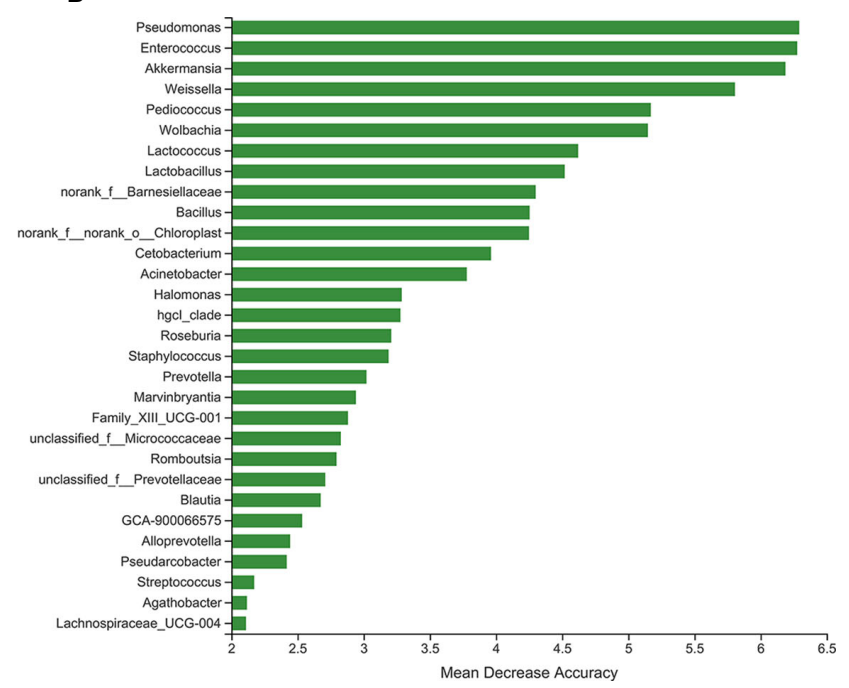

FIGURE 5 | The top 30 genera with great importance in distinguishing primary aldosteronism (PA) patients from (A) healthy controls and (B) primary hypertension patients. 


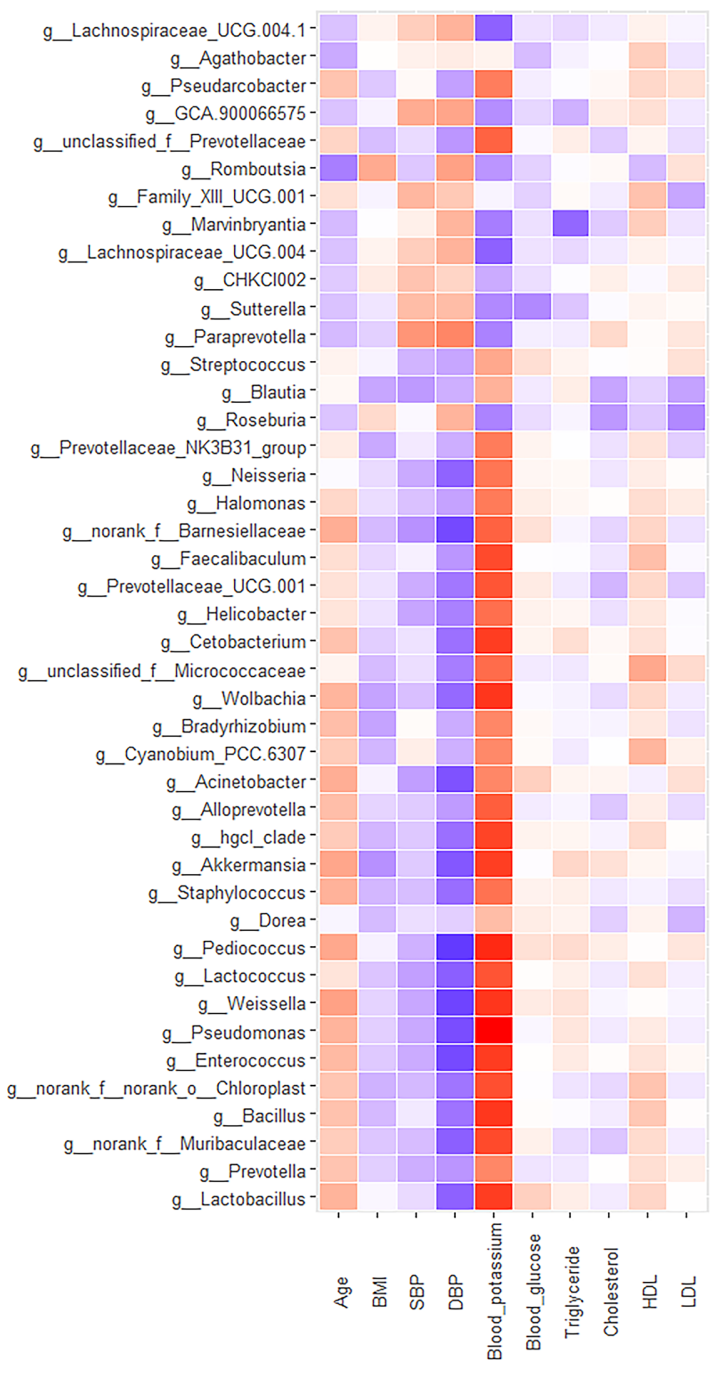

B
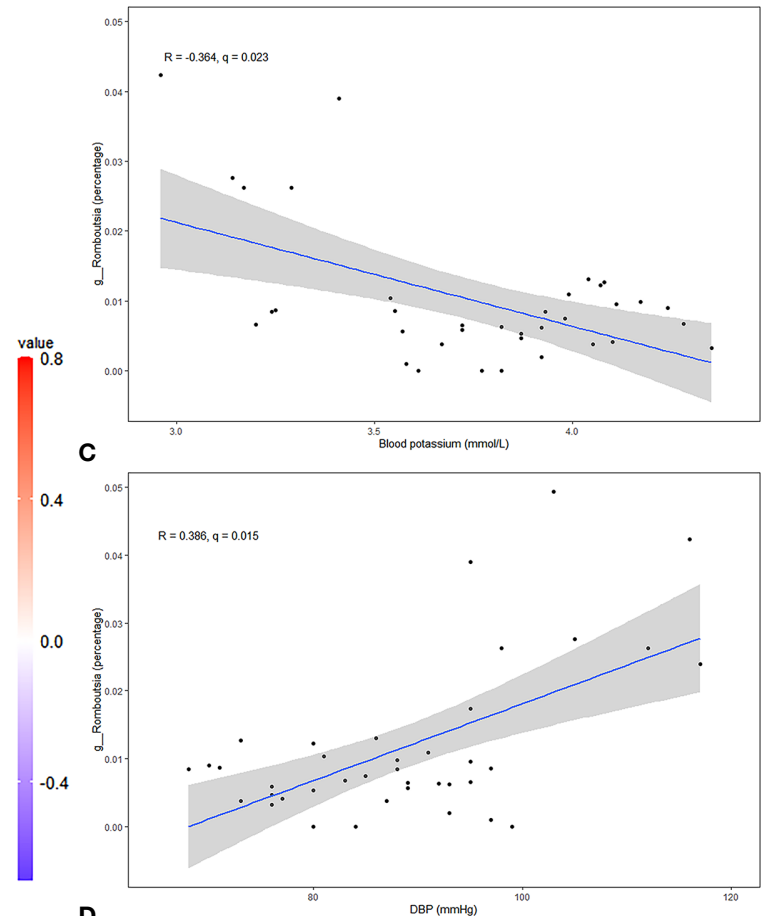

D

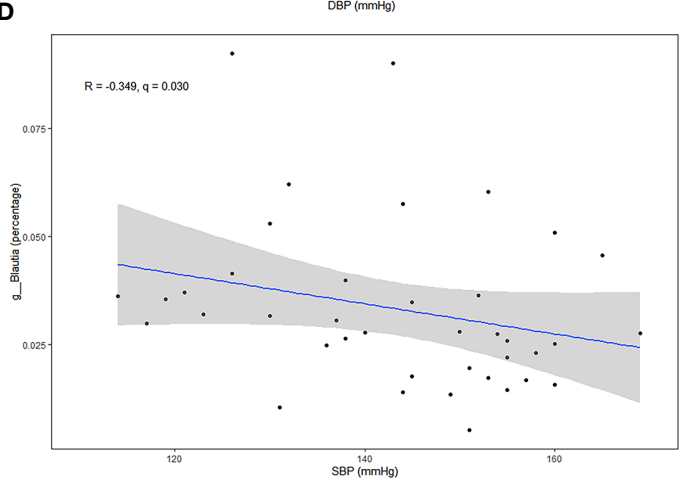

FIGURE 6 | Spearman correlation analysis of different bacteria [primary aldosteronism (PA) patients vs. primary hypertension patients] and clinical characteristics. (A) Heatmap showing the correlation between different bacteria and clinical characteristics. (B) Scatter plot graphs showing the correlation between blood potassium and Romboutsia. (C) Scatter plot graphs showing the correlation between diastolic blood pressure (DBP) and Romboutsia. (D) Scatter plot graphs showing the correlation between systolic blood pressure (SBP) and Blautia.

microbiota and its metabolic pathways of PA patients were also significantly different from those of healthy controls and primary hypertension patients.

We hypothesized that PA may contribute to the development of DM and obesity via gut microbiota. On the one hand, the alpha diversity of the gut microbiota in PA patients was lower than that in primary hypertension patients and healthy controls, which meant that the richness of the gut microbiota of PA patients was disturbed. Folz et al. reported that high-sodium and low-potassium intake could interact with gut microbiota to affect endocrine homeostasis (15). No study has clearly demonstrated the relationship between aldosterone and gut microbiota. It was reasonable to assume that $\mathrm{PA}$ is responsible for the changes in gut microbiota. On the other hand, the gut microbiota also affects DM and obesity by regulating inflammation, gut permeability, glucose metabolism, fatty acid oxidation, synthesis and energy expenditure, and the interaction of gut bacteria (6). The richness of gut microbiota in metabolic disease patients decreased (16). The Firmicutes/Bacteroidetes ratio was reported to be lower in individuals with obesity (17).

We found that the relative abundances of Prevotella, Blautia, Coprococcus, Anaerostipes, and Ruminococcus were lower in the gut microbiota of PA patients than in healthy controls and primary hypertension patients. All these bacteria could produce SCFAs (18-21). SCFAs, the energy sources of enterocytes, could maintain the intestinal epithelial barrier 
and decrease the permeability of the gut, circulating lipopolysaccharides, and systemic inflammation. SCFAs could also reduce inflammation via $\operatorname{GPR} 41 / 43(22,23)$. In addition, Lactobacillus was more abundant in the gut microbiota of healthy controls and primary hypertension patients than in PA patients. Several studies have already demonstrated that the administration of Lactobacillus could alleviate hyperglycemia in diabetic rats $(6,24,25)$. We also found that Lactococcus and Enterococcus were present in lower abundances in PA patients. Lilia et al. reported that consumption of fermented milk with Lactococcus lactis had a blood pressure-lowering effect on prehypertensive subjects (26). Anne et al. found that vertical sleeve gastrectomy reduced body mass and blood pressure and increased the relative abundance of Enterococcus in the gut microbiota of mice (27). In contrast, PA patients had more Megamonas, Sutterella, and Streptococcus than healthy controls and primary hypertension patients, genera that are associated with inflammation (28-30). Weissella and Akkermansia, which were present in lower abundances in PA patients than in primary hypertension patients, were shown to have antiinflammatory potential $(31,32)$. PA patients had a higher relative abundance of lipopolysaccharide biosynthesis in the gut microbiota than healthy controls. Lipopolysaccharides are bacterial surface glycolipids produced by gram-negative bacteria, which can induce inflammatory reactions (33). It was reported that inflammatory factors could alter glucose tolerance and insulin sensitivity (34). Individuals with obesity had more Megamonas (7). Compared with healthy controls and primary hypertension patients, PA patients had more pathways participating in sugar metabolism, such as starch and sucrose metabolism, fructose and mannose metabolism, amino sugar and nucleotide sugar metabolism, pentose and glucuronate interconversions, arginine and proline metabolism, and galactose metabolism. These factors may result in more glucose in the gut and absorption into the blood. In addition, some amino acid metabolism pathways were also significantly different between the PA and hypertension groups. For example, tryptophan metabolism was lower in PA patients. Tryptophan metabolism could produce some metabolites, such as indole-3-ethanol, indole-3-pyruvate, and indole-3-aldehyde, which are essential in maintaining intestinal barrier function (35). This evidence indicated that PA-associated dysbiosis of gut microbiota in SCFAs, sugar and amino acid metabolism, and inflammation were associated with metabolic disorders.

Previous studies regarded hypokalemia as a risk factor for DM. They thought that hypokalemia may decrease insulin secretion and induce insulin resistance, followed by dysbiosis of glucose metabolism (36). We found that Romboutisa and Bacteroides were more abundant in PA patients than in primary hypertension patients. Romboutisa was also negatively correlated with blood potassium. Some studies found that Romboutsia was positively associated with obesity and lipid metabolism $(37,38)$. Bacteroides was also found to be increased after the higher-fat diet intervention (39) and to be more abundant in patients with DM among the Chinese population (40). This evidence indicated that gut microbiota may also play an important role in hypokalemia-related disorders of lipid and glucose metabolism, a possibility that needs further exploration.

Our study first revealed the characteristics of the gut microbiota of PA patients and identified some bacteria and metabolic pathways that may be associated with DM and obesity. We believe that the knowledge gained from this study will be important in shaping the framework and platform for broader research on the gut microbiota of PA patients in the future.

The study showed that there was a significant difference in the mean age between PA patients and primary hypertension patients. Several studies have revealed that age is a factor influencing the gut microbiota (41-44). As people age, key changes in gut microbiota include compositional instability, reduced overall diversity, and an increase in proinflammatory opportunistic pathogens. In infants, Bifidobacterium is the most dominant genus in the gut microbiota. Biagi et al. examined the fecal microbiome of young adults (22-48 years old), elderly adults (65-75 years old), and semisupercentenarians (105109 years old) in an Italian population (45). They found that the fecal microbiota in all age groups was dominated by just three families, including Bacteroidaceae, Lachnospiraceae, and Ruminococcaceae. However, the relative abundance of bacteria belonging to these families decreased with age. An independent study of Chinese centenarians reported similar results (46). In addition, they both found that longevity increased microbial community richness and the abundance of subdominant but health-related bacterial genera and families, such as Oscillospira, Christensenellaceae, Akkermansia, and Bifidobacterium. The diversity of gut microbiota continues to increase until the age of 3 years, remains stable afterwards, and then declines during senescence (44). The gut microbiota of elderly people is reduced in beneficial microbes, such as SCFA producers, and enriched in proinflammatory microbes $(42,44)$.

This study had some limitations. First, almost all PA and primary hypertension patients had received different antihypertensive medications before recruitment. It was unclear whether these drugs affected the composition and metabolism of gut microbiota. Second, the effects of some factors on the gut microbiota could not be ignored, such as DM, aging, use of antihypertensive drugs, and salt intake (47). Third, the sample size of the study was relatively small and some characteristics were heterogeneous between groups, which may contribute to biases. Further case-control studies should have larger sample sizes; match factors that will potentially affect gut microbiota, such as age and dietary habits; and measure the 24-h urinary electrolytes to investigate the role of gut microbiota in metabolic disorders in primary aldosteronism patients and reduce analysis bias.

In conclusion, the alteration of gut microbiota in PA patients, especially bacteria and pathways involved in inflammation, SCFAs, and sugar metabolism, may be associated with chronic metabolic disorders. 


\section{DATA AVAILABILITY STATEMENT}

The datasets presented in this study can be found in online repositories. The names of the repository/repositories and accession number(s) can be found below: NCBI accession: PRJNA728662.

\section{ETHICS STATEMENT}

The studies involving human participants were reviewed and approved by the West China Hospital, Sichuan University Medical Research Ethics Committee. The patients/participants provided their written informed consent to participate in this study.

\section{AUTHOR CONTRIBUTIONS}

$\mathrm{YL}$ and QJ conceived and designed the study, collected and analyzed the data, and wrote the manuscript. ZL, SS, and JA analyzed the data. YZ and LZ reviewed and edited the

\section{REFERENCES}

1. Young WF, Calhoun DA, Lenders JWM, Michael S, Textor SC. Screening for Endocrine Hypertension: An Endocrine Society Scientific Statement. Endocrine Rev (2017) 2):103-22. doi: 10.1210/er.2017-00054

2. Akehi Y, Yanase T. High Prevalence of Diabetes in Patients With Primary Aldosteronism (PA) Associated With Subclinical Hypercortisolism and Prediabetes More Prevalent in Bilateral Than Unilateral PA: A Large. Multicenter Cohort Study Japan (2019) 42(5):938-45. doi: 10.2337/dc181293

3. Adolf C, Asbach E, Dietz AS, Lang K, Hahner S, Quinkler M, et al. Worsening of Lipid Metabolism After Successful Treatment of Primary Aldosteronism. Endocrine (2016) 54(1):198-205. doi: 10.1007/s12020-016-0983-9

4. Bothou C, Beuschlein F, Spyroglou A. Links Between Aldosterone Excess and Metabolic Complications: A Comprehensive Review. Diabetes Metab (2020) 46(1):1-7. doi: 10.1016/j.diabet.2019.02.003

5. Boulangé CL, Neves AL, Chilloux J, Nicholson JK, Dumas ME. Impact of the Gut Microbiota on Inflammation, Obesity, and Metabolic Disease. Genome Med (2016) 8(1):42. doi: 10.1186/s13073-016-0303-2

6. Gurung M, Li Z, You H, Rodrigues R, Jump DB, Morgun A, et al. Role of Gut Microbiota in Type 2 Diabetes Pathophysiology. EBioMedicine (2020) 51:102590. doi: 10.1016/j.ebiom.2019.11.051

7. Crovesy L, Masterson D, Rosado EL. Profile of the Gut Microbiota of Adults With Obesity: A Systematic Review. Eur J Clin Nutr (2020) 74(9):1251-62. doi: 10.1038/s41430-020-0607-6

8. Wu J, Wang K, Wang X, Pang Y, Jiang C. The Role of the Gut Microbiome and its Metabolites in Metabolic Diseases. Protein Cell (2021) 12(5):360-73. doi: 10.1007/s13238-020-00814-7

9. Magoč T, Salzberg SL. FLASH: Fast Length Adjustment of Short Reads to Improve Genome Assemblies. Bioinf (Oxford England) (2011) 27(21):295763. doi: 10.1093/bioinformatics/btr507

10. Chen S, Zhou Y, Chen Y, Gu J. Fastp: An Ultra-Fast All-in-One FASTQ Preprocessor. Bioinf (Oxford England) (2018) 34(17):i884-90. doi: 10.1093/ bioinformatics/bty560

11. Callahan BJ, McMurdie PJ, Rosen MJ, Han AW, Johnson AJ, Holmes SP. DADA2: High-Resolution Sample Inference From Illumina Amplicon Data. Nat Methods (2016) 13(7):581-3. doi: 10.1038/nmeth.3869

12. Bolyen E, Rideout JR, Dillon MR, Bokulich NA, Abnet CC, Al-Ghalith GA, et al. Reproducible, Interactive, Scalable and Extensible Microbiome Data manuscript. All authors contributed to the article and approved the submitted version.

\section{FUNDING}

This work was supported by 1.3.5 Project for Disciplines of Excellence, West China Hospital, Sichuan University (ZY2016104).

\section{ACKNOWLEDGMENTS}

We thank all the doctors, nurses, and laboratorian in the West China Hospital for their assistance and all the patients for their contribution to this study.

\section{SUPPLEMENTARY MATERIAL}

The Supplementary Material for this article can be found online at: https://www.frontiersin.org/articles/10.3389/fendo.2021. 667951/full\#supplementary-material

Science Using QIIME 2. Nat Biotechnol (2019) 37(8):852-7. doi: 10.1038/ s41587-019-0209-9

13. Segata N, Izard J, Waldron L, Gevers D, Miropolsky L, Garrett WS, et al. Metagenomic Biomarker Discovery and Explanation. Genome Biol (2011) 12 (6):R60. doi: 10.1186/gb-2011-12-6-r60

14. Douglas GM, Maffei VJ, Zaneveld JR, Yurgel SN, Brown JR, Taylor CM, et al. PICRUSt2 for Prediction of Metagenome Functions. Nat Biotechnol (2020) 38 (6):685-8. doi: 10.1038/s41587-020-0548-6

15. Folz J, Oh YT, Blaženović I, Richey J, Fiehn O, Youn JH. Interaction of Gut Microbiota and High-Sodium, Low-Potassium Diet in Altering Plasma Triglyceride Profiles Revealed by Lipidomics Analysis. Mol Nutr Food Res (2019) 63(24):e1900752. doi: 10.1002/mnfr.201900752

16. Sonnenburg ED, Smits SA, Tikhonov M, Higginbottom SK, Wingreen NS, Sonnenburg JL. Diet-Induced Extinctions in the Gut Microbiota Compound Over Generations. Nature (2016) 529(7585):212-5. doi: 10.1038/nature16504

17. Schwiertz A, Taras D, Schäfer K, Beijer S, Bos NA, Donus C, et al. Microbiota and SCFA in Lean and Overweight Healthy Subjects. Obes (Silver Spring Md) (2010) 18(1):190-5. doi: 10.1038/oby.2009.167

18. Mocanu V, Rajaruban S, Dang J, Kung JY, Deehan EC, Madsen KL. Repeated Fecal Microbial Transplantations and Antibiotic Pre-Treatment Are Linked to Improved Clinical Response and Remission in Inflammatory Bowel Disease: A Systematic Review and Pooled Proportion Meta-Analysis. J Clin Med (2021) 10(5):959. doi: 10.3390/jcm10050959

19. Li Y, Liu W, Xu ZZ, Xiao JX, Zong AZ, Qiu B, et al. Research on the Mechanism of Microwave-Toughened Starch on Glucolipid Metabolism in Mice. Food Funct (2020) 11(11):9789-800. doi: 10.1039/d0fo02093a

20. Jiang S, Xie S, Lv D, Wang P, He H, Zhang T, et al. Alteration of the Gut Microbiota in Chinese Population With Chronic Kidney Disease. Sci Rep (2017) 7(1):2870. doi: 10.1038/s41598-017-02989-2

21. Han SH, Yi J, Kim JH, Lee S, Moon HW. Composition of Gut Microbiota in Patients With Toxigenic Clostridioides (Clostridium) Difficile: Comparison Between Subgroups According to Clinical Criteria and Toxin Gene Load. PLoS One (2019) 14(2):e0212626. doi: 10.1371/journal.pone.0212626

22. Koh A, De Vadder F, Kovatcheva-Datchary P, Bäckhed F. From Dietary Fiber to Host Physiology: Short-Chain Fatty Acids as Key Bacterial Metabolites. Cell (2016) 165(6):1332-45. doi: 10.1016/j.cell.2016.05.041

23. Yao Y, Cai X, Fei W, Ye Y, Zhao M, Zheng C. The Role of Short-Chain Fatty Acids in Immunity, Inflammation and Metabolism. Crit Rev Food Sci Nutr (2020) 1-12. doi: 10.1080/10408398.2020.1854675 
24. Toejing P, Khat-Udomkiri N, Intakhad J, Sirilun S, Chaiyasut C. Putative Mechanisms Responsible for the Antihyperglycemic Action of Lactobacillus Paracasei HII01 in Experimental Type 2 Diabetic Rats. Nutrients (2020) 12 (10):3015. doi: 10.3390/nu12103015

25. Bauer PV, Duca FA, Waise TMZ, Dranse HJ, Rasmussen BA, Puri A, et al. Lactobacillus Gasseri in the Upper Small Intestine Impacts an ACSL3Dependent Fatty Acid-Sensing Pathway Regulating Whole-Body Glucose Homeostasis. Cell Metab (2018) 27(3):572-87.e6. doi: 10.1016/j.cmet. 2018.01.013

26. Beltrán-Barrientos LM, González-Córdova AF, Hernández-Mendoza A, Torres-Inguanzo EH, Astiazarán-García H, Esparza-Romero J, et al. Randomized Double-Blind Controlled Clinical Trial of the Blood PressureLowering Effect of Fermented Milk With Lactococcus Lactis: A Pilot Study. J dairy Sci (2018) 101(4):2819-25. doi: 10.3168/jds.2017-13189

27. McGavigan AK, Henseler ZM, Garibay D, Butler SD, Jayasinghe S, Ley RE, et al. Vertical Sleeve Gastrectomy Reduces Blood Pressure and Hypothalamic Endoplasmic Reticulum Stress in Mice. Dis Models Mech (2017) 10(3):235-43. doi: $10.1242 / \mathrm{dmm} .027474$

28. Ling Z, Jin C, Xie T, Cheng Y, Li L, Wu N. Alterations in the Fecal Microbiota of Patients With HIV-1 Infection: An Observational Study in A Chinese Population. Sci Rep (2016) 6:30673. doi: 10.1038/srep30673

29. Lan R, Wan Z, Xu Y, Wang Z, Fu S, Zhou Y, et al. Taurine Reprograms Mammary-Gland Metabolism and Alleviates Inflammation Induced by Streptococcus Uberis in Mice. Front Immunol (2021) 12:696101. doi: 10. 3389/fimmu.2021.696101

30. Hiippala K, Kainulainen V, Kalliomäki M, Arkkila P, Satokari R. Mucosal Prevalence and Interactions With the Epithelium Indicate Commensalism of Sutterella Spp. Front Microbiol (2016) 7:1706. doi: 10.3389/fmicb.2016.01706

31. Yu HS, Lee NK, Choi AJ, Choe JS, Bae CH, Paik HD. Anti-Inflammatory Potential of Probiotic Strain Weissella Cibaria JW15 Isolated From Kimchi Through Regulation of NF-Kb and MAPKs Pathways in LPS-Induced RAW 264.7 Cells. J Microbiol Biotechnol (2019) 29(7):1022-32. doi: 10.4014/ jmb.1903.03014

32. Grajeda-Iglesias C, Durand S, Daillère R, Iribarren K, Lemaitre F, Derosa L, et al. Oral Administration of Akkermansia Muciniphila Elevates Systemic Antiaging and Anticancer Metabolites. Aging (2021) 13(5):6375-405. doi: 10.18632/aging.202739

33. Candelli M, Franza L, Pignataro G, Ojetti V, Covino M, Piccioni A, et al. Interaction Between Lipopolysaccharide and Gut Microbiota in Inflammatory Bowel Diseases. Int J Mol Sci (2021) 22(12):6242. doi: 10. 3390/ijms22126242

34. Scheithauer TPM, Rampanelli E, Nieuwdorp M, Vallance BA, Verchere CB, van Raalte DH, et al. Gut Microbiota as a Trigger for Metabolic Inflammation in Obesity and Type 2 Diabetes. Front Immunol (2020) 11:571731. doi: 10.3389/fimmu.2020.571731

35. Scott SA, Fu J, Chang PV. Microbial Tryptophan Metabolites Regulate Gut Barrier Function via the Aryl Hydrocarbon Receptor. Proc Natl Acad Sci (2020) 117(32):19376-87. doi: 10.1073/pnas.2000047117

36. Liu Y, Zhou L, Liu Z, Ma Y, Lin L, Zhu Y, et al. Higher Blood Urea Nitrogen and Urinary Calcium: New Risk Factors for Diabetes Mellitus in Primary Aldosteronism Patients. Front Endocrinol (2020) 11:23. doi: 10.3389/ fendo.2020.00023
37. Vazquez-Moreno M, Perez-Herrera A, Locia-Morales D, Dizzel S, Meyre D. Association of Gut Microbiome With Fasting Triglycerides, Fasting Insulin and Obesity Status in Mexican Children. Pediatr Obes (2021) 16(5):e12748. doi: 10.1111/ijpo.12748

38. Li TT, Tong AJ, Liu YY, Huang ZR, Wan XZ, Pan YY, et al. Polyunsaturated Fatty Acids From Microalgae Spirulina Platensis Modulates Lipid Metabolism Disorders and Gut Microbiota in High-Fat Diet Rats. Food Chem Toxicol (2019) 131:110558. doi: 10.1016/j.fct.2019.06.005

39. Wan Y, Wang F, Yuan J, Li J, Jiang D, Zhang J, et al. Effects of Dietary Fat on Gut Microbiota and Faecal Metabolites, and Their Relationship With Cardiometabolic Risk Factors: A 6-Month Randomised Controlled-Feeding Trial. Gut (2019) 68(8):1417-29. doi: 10.1136/gutjnl-2018-317609

40. Qin J, Li Y, Cai Z, Li S, Zhu J, Zhang F, et al. A Metagenome-Wide Association Study of Gut Microbiota in Type 2 Diabetes. Nature (2012) 490(7418):55-60. doi: 10.1038/nature11450

41. Bosco N, Noti M. The Aging Gut Microbiome and its Impact on Host Immunity. Genes Immun (2021) 1-15. doi: 10.1038/s41435-021-00126-8

42. Ragonnaud E, Biragyn A. Gut Microbiota as the Key Controllers of "Healthy" Aging of Elderly People. Immun Ageing I A (2021) 18(1):2. doi: 10.1186/ s12979-020-00213-w

43. Yang J, Wu J, Li Y, Zhang Y, Cho WC, Ju X, et al. Gut Bacteria Formation and Influencing Factors. FEMS Microbiol Ecol (2021) 97(4):fiab043. doi: 10.1093/ femsec/fiab043

44. Walrath T, Dyamenahalli KU, Hulsebus HJ, McCullough RL, Idrovo JP, Boe $\mathrm{DM}$, et al. Age-Related Changes in Intestinal Immunity and the Microbiome. J Leukocyte Biol (2021) 109(6):1045-61. doi: 10.1002/jlb.3ri0620-405rr

45. Biagi E, Franceschi C, Rampelli S, Severgnini M, Ostan R, Turroni S, et al. Gut Microbiota and Extreme Longevity. Curr Biol CB (2016) 26(11):1480-5. doi: 10.1016/j.cub.2016.04.016

46. Wang N, Li R, Lin H, Fu C, Wang X, Zhang Y, et al. Enriched Taxa Were Found Among the Gut Microbiota of Centenarians in East China. PLoS One (2019) 14(10):e0222763. doi: 10.1371/journal.pone.0222763

47. Wilck N, Matus MG, Kearney SM, Olesen SW, Forslund K, Bartolomaeus H, et al. Salt-Responsive Gut Commensal Modulates $\mathrm{T}(\mathrm{H}) 17$ Axis and Disease. Nature (2017) 551(7682):585-9. doi: 10.1038/nature24628

Conflict of Interest: The authors declare that the research was conducted in the absence of any commercial or financial relationships that could be construed as a potential conflict of interest.

Publisher's Note: All claims expressed in this article are solely those of the authors and do not necessarily represent those of their affiliated organizations, or those of the publisher, the editors and the reviewers. Any product that may be evaluated in this article, or claim that may be made by its manufacturer, is not guaranteed or endorsed by the publisher.

Copyright (c) 2021 Liu, Jiang, Liu, Shen, Ai, Zhu and Zhou. This is an open-access article distributed under the terms of the Creative Commons Attribution License (CC BY). The use, distribution or reproduction in other forums is permitted, provided the original author(s) and the copyright owner(s) are credited and that the original publication in this journal is cited, in accordance with accepted academic practice. No use, distribution or reproduction is permitted which does not comply with these terms. 